\title{
Musicoterapia e autismo em uma perspectiva comportamental
}

\author{
Music therapy and autism in a behavioral perspective
}

\author{
La musicoterapia y el autismo en una perspectiva \\ conductual
}

\author{
Sarah Caroline Jeronimo da Silva ${ }^{1}$, Rita de Cássia dos Reis Moura² \\ 1.Musicoterapeuta. Especialista em Musicoterapia Aplicada, Departamento de Música, Faculdade Santa \\ Marcelina, São Paulo-SP, Brasil. Orcid: https://orcid.org/0000-0001-6315-2915 \\ 2.Doutorado pela Universidade Federal de São Paulo (UNIFESP), Coordenadora e Professora do curso de \\ Pós-Graduação em Musicoterapia Aplicada, Departamento de Música, Faculdade Santa Marcelina, São \\ Paulo-SP, Brasil. Orcid: https://orcid.org/0000-0003-0150-7586
}

\section{Resumo}

Introdução. $O$ autismo é alvo de investigações de muitos pesquisadores. Até o momento não se encontram biomarcadores claros sobre sua fisiopatologia e, por consequência, o diagnóstico tem como base critérios comportamentais. A musicoterapia vem crescendo no campo de tratamento do autismo, uma vez que estes indivíduos apresentam grande interesse pelos estímulos musicais. Objetivo. Identificar como a musicoterapia pode beneficiar, em uma perspectiva comportamental, o tratamento do autismo. Método. Foi realizada revisão bibliográfica nas bases de dados PubMed, Google Scholar e Scielo com os descritores em inglês, português e espanhol contendo "Musicoterapia e Autismo", "Musicoterapia Comportamental e Autismo" e "Musicoterapia Comportamental". Resultados. Foram encontrados 16 artigos que atenderam aos critérios de inclusão deste estudo, divididos em duas categorias: (tipo de intervenção e revisão bibliográfica) para facilitar a apresentação dos achados. Conclusão. A musicoterapia apresenta resultados relevantes para a redução de comportamentos estereotipados e aumento de habilidades sociais e comunicação em pessoas com autismo, tendo como principal abordagem utilizada a musicoterapia criativa e comportamental, buscando alcançar resultados não-musicais apoiados em evidências neurocientíficas. Apesar dos resultados positivos, nota-se uma escassez de trabalhos com amostras maiores e melhores com evidências científicas.

Unitermos. Musicoterapia e autismo; Musicoterapia Comportamental; Musicoterapia Improvisacional; Musicoterapia Criativa; Musicoterapia Baseada em Evidências

\begin{abstract}
Introduction. Autism is the subject of investigation by many researchers. So far, there are no clear biomarkers about its pathophysiology and, consequently, the diagnosis is based on behavioral criteria. Music therapy has been growing in the field of autism treatment since these individuals are very interested in musical stimuli. Objectives. To identify how music therapy can benefit, in a behavioral perspective, the treatment of autism. Method: a bibliographic review was carried out in the PubMed, Google Scholar and Scielo databases with the descriptors in English, Portuguese, and Spanish containing "Music Therapy and Autism", "Behavioral Music Therapy and Autism" and "Behavioral Music Therapy". Results. 16 articles were found that met the inclusion criteria of this study, divided into two categories: (type of intervention and bibliographic review) to facilitate the presentation of the findings. Conclusions. Music therapy presents relevant results for reducing stereotyped behaviors and increasing social and communication skills in people with autism, having creative and behavioral music therapy as the main approach, seeking to achieve non-musical results supported by neuroscientific evidence. Despite the positive results, there is a scarcity of studies with larger and better samples with scientific evidence.
\end{abstract}

Keywords. Music therapy and autism; Behavioral Music Therapy; Improvisational Music Therapy; Creative Music Therapy; Evidence-Based Music Therapy 


\section{Resumen}

Introducción. El autismo es objeto de investigación por parte de muchos investigadores. Hasta el momento, no existen biomarcadores claros sobre su fisiopatología y, en consecuencia, el diagnóstico se basa en criterios conductuales. La musicoterapia ha ido creciendo en el campo del tratamiento del autismo, ya que estos individuos están muy interesados en los estímulos musicales. Objetives. Identificar cómo la musicoterapia puede beneficiar, desde una perspectiva conductual, el tratamiento del autismo. Método. Se realizó una revisión bibliográfica en las bases de datos PubMed, Google Scholar y Scielo con los descriptores en inglés, portugués y español que contienen "Musicoterapia y autismo", "Musicoterapia conductual y autismo" y "Musicoterapia conductual". Resultados. Se encontraron 16 artículos que cumplieron con los criterios de inclusión de este estudio, divididos en dos categorías: (tipo de intervención y revisión bibliográfica) para facilitar la presentación de los hallazgos. Conclusiones: la musicoterapia presenta resultados relevantes para la reducción de conductas estereotipadas y el aumento de las habilidades sociales y comunicativas en personas con autismo, teniendo como enfoque principal la musicoterapia creativa y conductual, buscando lograr resultados no musicales apoyados en evidencia neurocientífica. A pesar de los resultados positivos, hay escasez de estudios con muestras más grandes y mejores con evidencia científica.

Palabras clave. Musicoterapia y autismo; Musicoterapia conductual; Musicoterapia de improvisación; Musicoterapia creativa; Musicoterapia basada en evidencias

Trabalho realizado na Faculdade Santa Marcelina, São Paulo-SP, Brasil.

\section{INTRODUÇÃO}

A fisiopatologia e etiologia do Transtorno do Espectro Autista (TEA) está em constante estudo, não se encontra até o momento biomarcadores claros sobre sua fisiopatologia e por consequência o diagnóstico tem como base critérios comportamentais ${ }^{1}$. O Manual Diagnóstico e Estatístico de Transtornos Mentais (DSM-5) classifica os sintomas do autismo em dois domínios, sendo o primeiro voltado a comunicação social e interação, incluindo déficits de comunicação verbal e não verbal, e os comportamentos repetitivos como segundo domínio. Os sintomas variam entre as crianças, de leves a graves e na mesma pessoa em diferentes idades ${ }^{2}$. Os dados estatísticos dos Estados Unidos das Américas (EUA) em março de 2020, apresentam uma prevalência de 1 autista para cada 54 crianças de 8 anos. A 
Organização Mundial de Saúde (OMS) aponta a prevalência de pelo menos $1 \%$ da população mundial com autismo. No Brasil estima-se que os dados sejam de 1 autista a cada 100 pessoas $^{3}$. Esses dados são incertos, uma vez que não existem estudos estatísticos conclusivos no Brasil e no mundo para dar o panorama real ${ }^{3,4}$.

Apesar de o autismo ser uma doença crônica e não progressiva, existem programas de ensino especial e suporte que promovem, muitas vezes, uma melhora marcante no comportamento, considerando grau de comprometimento, comorbidades, situação familiar e social, saúde e demais fatores que o cercam. Entre as terapias mencionadas pelo Ministério da Saúde para o tratamento do autismo, incluem a Integração Sensorial, Fonoaudiologia, Terapia Ocupacional, Fisioterapia, Análise do Comportamento Aplicada (Applied Behavior Analysis - ABA) e Musicoterapia².

Evidências acerca da Musicoterapia na reabilitação neurológica apontam que os componentes da música como ritmo, melodia, harmonia, timbre, forma e dinâmica podem estimular processos cognitivos, sensório motores e afetivos complexos no cérebro, generalizando e transferindo estas funções para fins terapêuticos não-musicais e modulando alterações comportamentais e funcionais ${ }^{5}$.

O objetivo deste estudo foi elencar artigos que versem sobre a Musicoterapia sob uma ótica comportamental no tratamento de crianças com autismo, a fim de conhecer as principais técnicas e métodos utilizados para alcançar melhora nos comportamentos presentes no TEA. 


\section{MÉTODO}

Foi realizada revisão de literatura com busca nas bases de dados Google Scholar, PubMed e Scielo, utilizando-se os descritores em inglês, português e espanhol: "Musicoterapia e Autismo", "Musicoterapia Comportamental e Autismo", "Musicoterapia Comportamental", no período de 2016 a 2020.

Foram incluídos artigos que abordam a temática da Musicoterapia no tratamento comportamental de pessoas com Autismo. Foram excluídos artigos que fugiram da temática deste trabalho, como o uso terapêutico da música onde a proposta principal não era a musicoterapia, artigos incompletos, resenhas, livros e teses.

\section{RESULTADOS}

Foram encontrados 476 estudos, destes apenas 16 atenderam aos critérios de inclusão, sendo que destes quatro são brasileiros. Dividimos os artigos em duas categorias para melhor compreensão e apresentação dos achados, são elas:

1. Categoria Intervenção - com 6 artigos que apresentaram distintos delineamentos de estudo: ensaios randomizados, estudo de caso e estudo transversal. As intervenções apresentaram os seguintes objetivos: melhora na comunicação (3); nas habilidades sociais (2) e o outro estudo que se ocupou no levantamento das práticas mais utilizadas por musicoterapeutas americanos em pacientes com autismo6-11. 
2. Categoria Revisão - com 8 artigos, dentre eles 2 relacionados aos circuitos neurais e reações fisiológicas, percepção, recompensa e emoção; outros 2 artigos direcionados às habilidades sociais; um sobre improvisação como forma de intervenção e 3 de revisões sistemáticas. Estas divisões serão apresentadas nos Quadros 1 e $2^{12-19}$.

3. Outras Categorias - Dois artigos não foram incluídos nessa classificação, sendo um deles sobre os biomarcadores envolvidos no autismo e a influência da música sobre os mesmos; e o outro apresenta um método de avaliação por tecnologia computadorizada das preferências musicais das crianças com TEA 20,21 .

\section{Categoria Intervenção}

\section{Comunicação}

Sharda et al. ${ }^{6}$ realizaram um estudo em crianças em idade escolar (6 a 12 anos). As intervenções foram estruturadas a partir da abordagem centrada na criança. Os resultados avaliados através de ressonância magnética funcional em estado de repouso (Resting state functional magnetic resonance imaging - rsfMRI) apontam ganhos de comunicação funcional, o grupo que recebeu a musicoterapia aumentou a conectividade funcional entre o córtex auditivo primário bilateral e as regiões subcorticais e motoras (muitas vezes reduzidas no TEA) e a excesso de conectividade entre as áreas auditivas e de associação visual. Os autores ressaltam melhorias nas habilidades de comunicação das crianças após a musicoterapia. 


\begin{tabular}{|c|c|c|}
\hline $\begin{array}{c}\text { Autor/ } \\
\text { data }\end{array}$ & Objetivo/Metodologia & Resultados \\
\hline $\begin{array}{c}\text { Sharda et al. } \\
\text { 2018, } \\
\text { Canadá6 }\end{array}$ & $\begin{array}{l}\text { Ao investigar se a intervenção baseada } \\
\text { em música pode melhorar a } \\
\text { conectividade cerebral funcional, } 51 \\
\text { crianças de } 6 \text { a } 12 \text { anos com autismo } \\
\text { foram randomizadas para receber } \\
\text { intervenção musical improvisada, sendo } \\
\text { avaliadas antes e após as intervenções } \\
\text { com Imagem de ressonância magnética } \\
\text { funcional em estado de repouso (Resting } \\
\text { state functional magnetic resonance } \\
\text { imaging - rsfMRI) e protocolos para } \\
\text { profissionais e família. }\end{array}$ & $\begin{array}{l}8 \quad \text { a } 12 \text { semanas de } \\
\text { intervenção de musicoterapia } \\
\text { podem melhorar a } \\
\text { comunicação social, qualidade } \\
\text { de vida familiar e a } \\
\text { conectividade intrínseca do } \\
\text { cérebro em crianças em idade } \\
\text { escolar. }\end{array}$ \\
\hline $\begin{array}{c}\text { Garcia 2018, } \\
\text { Espanha7 }^{2}\end{array}$ & $\begin{array}{l}\text { Estudo de caso para apresentar os } \\
\text { resultados da musicoterapia com um } \\
\text { menino de } 5 \text { anos diagnosticado com } \\
\text { TEA. As sessões ocorreram } \\
\text { semanalmente por três meses, tendo } \\
\text { como intervenção a Musicoterapia } \\
\text { Improvisacional baseada nas } 64 \text { técnicas } \\
\text { de K. Bruscia. }\end{array}$ & $\begin{array}{l}\text { Através da musicoterapia } \\
\text { improvisacional, foi possível } \\
\text { alcançar melhoras na atenção, } \\
\text { ampliação de respostas vocais } \\
\text { e redirecionamentor de } \\
\text { estereotipias } \\
\text { movimentos funcionais com } \\
\text { instrumentos. }\end{array}$ \\
\hline $\begin{array}{c}\text { Novenia } \\
\text { 2019, } \\
\text { Indonésia8 }^{8}\end{array}$ & $\begin{array}{l}\text { Compreender como o uso da } \\
\text { musicoterapia melhora as habilidades de } \\
\text { comunicação não verbal para crianças } \\
\text { com autismo. Quatro crianças } \\
\text { diagnosticadas com autismo com idades } \\
\text { de } 6 \text { a } 11 \text { anos receberam durante três } \\
\text { meses intervenções musicoterapêuticas, } \\
\text { baseadas no método de Musicoterapia } \\
\text { Improvisacional. }\end{array}$ & $\begin{array}{l}\text { Sessões de musicoterapia } \\
\text { improvisacional com crianças } \\
\text { diagnosticadas com TEA em } \\
\text { idade escolar demonstram que } \\
\text { o aprimoramento nas } \\
\text { habilidades de comunicação e } \\
\text { na interação de crianças com } \\
\text { autismo pode ser alcançada } \\
\text { em até cinco sessões de } \\
\text { musicoterapia. }\end{array}$ \\
\hline $\begin{array}{l}\text { Khanzadeh } \\
\text { et al. 2017, } \\
\text { Irã9 }\end{array}$ & $\begin{array}{l}\text { Investigar o efeito da Musicoterapia } \\
\text { Orff-Schulwerk e Terapia Lúdica no } \\
\text { aumento de habilidades sociais e } \\
\text { diminuição de estereotipias em crianças } \\
\text { com TEA. Trinta crianças de } 6 \text { a } 12 \text { anos } \\
\text { com autismo foram randomizadas para } \\
\text { receber } 15 \text { sessões de musicoterapia } \\
\text { associada à terapia lúdica. }\end{array}$ & $\begin{array}{l}\text { A musicoterapia em conjunto } \\
\text { com a terapia lúdica } \\
\text { apresentou resultados } \\
\text { significativos na melhoria das } \\
\text { habilidades sociais, diminuição } \\
\text { de estereotipias e } \\
\text { comportamentos disruptivos. }\end{array}$ \\
\hline $\begin{array}{c}\text { Pater et al. } \\
2020, \\
\text { Holanda }^{10}\end{array}$ & $\begin{array}{l}\text { Estudo de caso sobre a contribuição da } \\
\text { musicoterapia no desenvolvimento de } \\
\text { habilidades sociais de uma criança com } \\
\text { autismo. O paciente recebeu } 20 \\
\text { semanas de } \\
\text { improvisacional. }\end{array}$ & $\begin{array}{l}\text { Estudo de caso que } \\
\text { apresentou resultados } \\
\text { positivos nas habilidades } \\
\text { sociais após } 20 \text { sessões de } \\
\text { musicoterapia, apresentando } \\
\text { ganhos residuais após } 6 \\
\text { meses de encerramento das } \\
\text { intervenções. }\end{array}$ \\
\hline $\begin{array}{l}\text { Bilgehan } \\
\text { 2017, } \\
\text { Turquia11 }^{11}\end{array}$ & $\begin{array}{l}\text { Análise das abordagens preferidas pelos } \\
\text { musicoterapeutas dos EUA para o } \\
\text { tratamento de crianças com autismo, } \\
\text { através de um questionário enviado para } \\
\text { os musicoterapeutas, a fim de } \\
\text { compreender como atuam e quais são as } \\
\text { abordagens preferidas para tratar } \\
\text { crianças com autismo. }\end{array}$ & $\begin{array}{l}\text { Entre todas as categorias } \\
\text { questionadas, os resultados } \\
\text { apresentaram a Musicoterapia } \\
\text { Criativa, } \\
\text { Comportamental } \\
\text { Musicoterapia de Integração } \\
\text { Sensorial como as três } \\
\text { principais abordagens com } \\
\text { eficácia para o tratamento de } \\
\text { crianças com autismo }\end{array}$ \\
\hline
\end{tabular}




\begin{tabular}{|c|c|c|}
\hline $\begin{array}{c}\text { Autor } \\
\text { data }\end{array}$ & Objetivo/Metodologia & Resultados \\
\hline $\begin{array}{l}\text { Quintin } \\
\text { 2019, } \\
\text { Canadá12 }\end{array}$ & $\begin{array}{l}\text { Avaliação de artigos sobre a percepção } \\
\text { musical, ativação de circuitos neurais de } \\
\text { recompensa e resposta emocional, bem } \\
\text { como a utilização da mesma para a } \\
\text { avaliação destas respostas fisiológicas em } \\
\text { autistas. }\end{array}$ & $\begin{array}{l}\text { A música ativa os sistemas de } \\
\text { recompensa e facilita o processo de } \\
\text { neuroplasticidade cerebral, podendo } \\
\text { ser utilizada para avaliar estes } \\
\text { aspectos em indivíduos com TEA. }\end{array}$ \\
\hline $\begin{array}{c}\text { Bharahi et } \\
\text { al. } 2019, \\
\text { Índia }^{13}\end{array}$ & $\begin{array}{l}\text { Discutir sobre a intervenção musical e } \\
\text { arrastamento rítmico no aumento da } \\
\text { plasticidade cortical em indivíduos com } \\
\text { TEA. Foi discutido o papel da indicação } \\
\text { rítmica na regulação sensório-motora em } \\
\text { indivíduos com autismo. }\end{array}$ & $\begin{array}{l}\text { O arrastamento rítmico desempenha } \\
\text { um papel importante em alterações } \\
\text { neurofisiológicas, podendo facilitar } \\
\text { processos de estimulação da fala, } \\
\text { motricidade e linguagem no TEA. }\end{array}$ \\
\hline $\begin{array}{l}\text { Fleury et } \\
\text { al. } 2016, \\
\text { Brasil }^{14}\end{array}$ & $\begin{array}{l}\text { Revisão de estudos sobre abordagens e } \\
\text { métodos musicoterapêuticos que } \\
\text { apresentam resultados positivos para } \\
\text { estimular a interação e habilidades sociais } \\
\text { de pessoas com autismo. }\end{array}$ & $\begin{array}{l}\text { A musicoterapia improvisacional é } \\
\text { apresentada como a principal técnica } \\
\text { musicoterapêutica nas intervenções } \\
\text { com autistas, facilitando a interação e } \\
\text { habilidades sociais. }\end{array}$ \\
\hline $\begin{array}{l}\text { LaGasse } \\
2017 \\
\text { EUA }^{15}\end{array}$ & $\begin{array}{l}\text { Apresentação de dados sobre a } \\
\text { musicoterapia no autismo e as } \\
\text { possibilidades de avaliação utilizando os } \\
\text { parâmetros musicais como potência para } \\
\text { prognóstico terapêutico. }\end{array}$ & $\begin{array}{l}\text { A música fornece medidas de avaliação } \\
\text { do funcionamento social do indivíduo } \\
\text { na sessão de musicoterapia e } \\
\text { generalização para ambientes não- } \\
\text { musicais, se tornando um dado } \\
\text { importante a ser considerado pelos } \\
\text { musicoterapeutas. }\end{array}$ \\
\hline $\begin{array}{l}\text { Suvini } \\
\text { 2019, } \\
\text { Itália16 }^{16}\end{array}$ & $\begin{array}{l}\text { Avaliação de artigos que explanam sobre } \\
\text { os aspectos da musicoterapia } \\
\text { improvisacional no tratamento de } \\
\text { crianças com autismo. }\end{array}$ & $\begin{array}{l}\text { A musicoterapia improvisacional torna } \\
\text { possível o diálogo com sons devido sua } \\
\text { baixa organização formal e atividades } \\
\text { musicais baseadas em imitação e } \\
\text { sincronização, podendo ser } \\
\text { particularmente úteis no tratamento } \\
\text { do autismo }\end{array}$ \\
\hline $\begin{array}{c}\text { Maw et al. } \\
2018 \\
\text { Japão }^{17}\end{array}$ & $\begin{array}{l}\text { Revisão sistemática sobre a eficácia da } \\
\text { Musicoterapia no tratamento de crianças } \\
\text { com autismo em idade pré-escolar. }\end{array}$ & $\begin{array}{l}\text { A Musicoterapia apresentou os } \\
\text { maiores efeitos para melhorar a } \\
\text { comunicação e as interações sociais } \\
\text { das crianças autistas, mas parece } \\
\text { pouco recomendada como tratamento } \\
\text { primário devido à falta de evidências } \\
\text { científicas adequadas. }\end{array}$ \\
\hline $\begin{array}{c}\text { Lyra et al. } \\
2017 \\
\text { Brasil }^{18}\end{array}$ & $\begin{array}{l}\text { Revisão sistemática e meta-análise de } \\
\text { resultados presentes sobre intervenções } \\
\text { farmacológicas e não farmacológicas } \\
\text { existentes publicadas na base de dados } \\
\text { Cochrane. }\end{array}$ & $\begin{array}{l}\text { A musicoterapia aparece entre as } \\
\text { intervenções não farmacológicas que } \\
\text { parecem ser benéficas no tratamento } \\
\text { do autismo, porém as revisões } \\
\text { sistemáticas sobre o tema mostram } \\
\text { baixa qualidade de evidências. }\end{array}$ \\
\hline $\begin{array}{l}\text { Alves et } \\
\text { al. } 2018, \\
\text { Brasil }^{19}\end{array}$ & $\begin{array}{l}\text { Comparar publicações nacionais e } \\
\text { internacionais a fim de identificar os tipos } \\
\text { de pesquisas trabalhadas sob a temática } \\
\text { "Musicoterapia e Autismo" no período de } \\
2016 \text { a } 2017 \text {. }\end{array}$ & $\begin{array}{l}\text { Foram analisados } 24 \text { artigos, sendo } 14 \\
\text { com intervenção e } 10 \text { sem intervenção } \\
\text { musicoterapêutica. Apesar da } \\
\text { relevância do tema para a área, } \\
\text { existem poucos achados sobre } \\
\text { Musicoterapia e Autismo no mundo. }\end{array}$ \\
\hline
\end{tabular}


A musicoterapia improvisacional foi apontada no estudo de caso de Garcia7, como técnica utilizada para a evocação de respostas vocais e instrumentais, facilitando, através de estimulação rítmica, a interação comunicativa entre criança e terapeuta, bem como a estimulação pré-verbal vocal como ponto de partida para aumentar sua intenção de habilidades comunicativas. A musicoterapia criativa facilitou a diminuição de momentos de desatenção do paciente, ampliando as respostas vocais quando estas foram integradas à improvisação e através de técnicas como a imitação e sincronização. O ritmo facilitou a diminuição de estereotipias manuais através do uso de objetos intermediários como baquetas.

No estudo realizado por Novenia ${ }^{8}$ são apresentados quatro estágios de desenvolvimento da comunicação para crianças com autismo: o primeiro é a "agenda própria", o segundo é o "estágio de solicitante"; o terceiro é o estágio "inicial da comunicação" e a última etapa é classificada como o nível "parceiro". A autora frisa que cada criança tem um tempo diferente para atingir esse objetivo de longo prazo. Com estes dados, a autora realizou intervenções musicoterapêuticas na abordagem improvisacional com quatro crianças. $O$ estudo conclui que o aprimoramento nas habilidades de comunicação e interação das crianças com autismo pode ser alcançada em até cinco sessões de musicoterapia. A autora destaca que a melhoria também está baseada no vínculo terapêutico entre paciente e terapeuta. 


\section{Habilidades sociais}

$\mathrm{Na}$ pesquisa experimental de Khanzedeh et al. ${ }^{9}$, baseada no método Musicoterapia Orff-Schulwerk, foram utilizadas as técnicas de Musicoterapia ativa e passiva para desenvolver o engajamento através de sons, palavras e sílabas acompanhadas de músicas favoritas e atraentes para as crianças e, posteriormente, alguns brinquedos e jogos foram gradualmente utilizados. Os resultados apresentaram redução significativa nos comportamentos estereotipados, na agressividade, timidez, comportamentos disruptivos e aumento das habilidades sociais de relevância no grupo experimental em comparação ao grupo controle.

Pater et al. ${ }^{10}$ utilizaram a musicoterapia ativa baseada em improvisação para desenvolver o foco na tarefa, melhorar habilidades e manter interação e comunicação em um paciente de 10 anos com autismo. Evolução foi monitorada através de questionários semanais. O resultado apresentou progresso significativo no contato visual, concentração, lidar com as mudanças, comunicação verbal e atenção conjunta. Apesar de significantes benefícios os autores indicam a falta de pesquisas estruturadas e fundamentação sobre a prática dos musicoterapeutas, pois muitas vezes há indícios que os musicoterapeutas seguem suas intuições e não existe uma estruturação clara de base teórica. 


\section{Abordagens mais utilizadas em musicoterapia}

Bilgehan ${ }^{11}$ em questionário estruturado e direcionado a musicoterapeutas americanos analisou as abordagens preferidas por esses profissionais para o tratamento de crianças com autismo. Foram citadas 17 abordagens de musicoterapia de acordo com a literatura relacionada, no entanto apenas as três mais referendadas foram elencadas como as preferidas e mais eficazes: Musicoterapia Comportamental, Musicoterapia de Integração Sensorial e Musicoterapia Criativa (improvisacional).

A Musicoterapia Comportamental, baseada no Behaviorismo, tem como finalidade observar comportamentos de um cliente e redirecionar os comportamentos indesejáveis específicos para mudança. São utilizadas técnicas de condicionamento operante ou clássico, utilizando a música como reforçadora para promover a mudança, uma vez que é considerada um forte estímulo motivador e desencadeia recompensa desejável para criar ou manter comportamentos. Embora a abordagem comportamental tenha sido relatada como a mais preferida, nas opiniões quanto sua eficácia foi identificada como a terceira abordagem eficaz ${ }^{11}$.

A Musicoterapia de Integração Sensorial é considerada como um conjunto de técnicas de integração sensorial no processo terapêutico, uma vez que utiliza a música, por meio de seus elementos, como um estímulo multidimensional e multissensorial nos processos de integração sensorial, que se dá pelo processo do sistema de receber e organizar 
bilhões de bits de entrada sensorial descoordenada. Intervenções como a internalização do ritmo, respostas adaptativas ao ambiente de estímulos auditivos e visuais, integração e discriminação auditiva, integração auditivofísica, planejamento motor, ações vestibulares, corpo coordenação no espaço, integração auditivo-visual, ritmo de movimento corporal, estabelecimento de limites e redirecionamento comportamental, criatividade, fala e linguagem são utilizadas. Foi escolhida como a segunda abordagem preferida e a mais eficaz para o tratamento do autismo ${ }^{11}$.

Musicoterapia Criativa assinalada como a segunda mais eficaz e terceira preferida entre os musicoterapeutas americanos, se trata de uma abordagem de improvisação musical desenvolvida por Paul Nordoff, um compositorpianista americano e Clive Robbins, um educador especial com formação britânica. O objetivo principal da improvisação é envolver o cliente na produção musical, onde suas respostas estão no centro da experiência terapêutica. Entre os objetivos clínicos abordados dentro da Musicoterapia Criativa se encontram a memória, empatia, diminuição de estereotipias, comunicação, autocontrole, interação social, criatividade $^{11}$.

\section{Categoria Revisão}

\section{Circuitos neurais e música}

A revisão de Quintin ${ }^{12}$ apresentou evidências que apontam a música como abordagem para avaliar recompensa e resposta emocional como uma poderosa 
ferramenta de intervenção para indivíduos com TEA, sendo que ouvir músicas emocionalmente intensas e agradáveis pode provocar uma excitação no sistema nervoso autônomo, incluindo o aumento da frequência cardíaca e profundidade na respiração, bem como o sistema de recompensa dopaminérgica para estímulos que evocam prazer e emoções intensas. Conclui-se que a musicoterapia pode ter um impacto positivo nas habilidades sociais e comunicação em crianças e adolescentes com autismo, bem como estes objetivos podem ser trabalhados em conjunto com a educação musical, que tem um potencial de melhorar a memória de trabalho, funcionamento executivo e habilidades de linguagem. Ambas as intervenções podem ganhar muito com sua fusão.

Bharathi et al. ${ }^{13}$ indicam existência de $70 \%$ disfunção sensorial e déficits nos desenvolvimentos motores orais nos indivíduos autistas, relacionados com a produção da fala, fluência e clareza, porém há uma escassez de intervenções que visam as características motora-orais no TEA. Crianças com dificuldades motoras tendem a permanecer não verbais ou apresentar problemas com fala e linguagem expressiva.

Audição musical e padrões rítmicos melhoram a atenção em crianças com TEA, indicando aprimoramento do sinal de integração sensorial. São apresentadas quatro intervenções principais de musicoterapia para o TEA: mapeamento motor auditivo, terapia de entonação melódica, musicoterapia improvisacional e treinamento rítmico. Os autores indicam que $o$ potencial do treinamento rítmico melhora $o$ 
funcionamento sensório-motor em TEA e tem ganhado impulso para o tratamento. Para esses autores os defeitos de desenvolvimento no tronco cerebral e cerebelo na fase intrauterina podem levar a déficits na percepção sensorial no TEA. Trevarthen et al. ${ }^{20}$ sugeriram que o ritmo e sincronia desorganizados em bebês podem ser os primeiros sinais de TEA e síndrome de Rett. A ritmicidade desempenha um papel vital no desenvolvimento e o tempo é fundamental no controle motor e funções cognitivas. O ritmo também incorpora a percepção sensorial e o arrastamento motor, o que resulta em funções cognitivas e adaptações motoras complexas ${ }^{13}$.

O "Rhythmic Entrainment" (arrastamento rítmico) é a primeira teoria motora para a função do ritmo auditivo e da música na terapia. O arrastamento rítmico tem papel importante em alterações neurofisiológicas, envolvendo diversas áreas cerebrais e melhorando a plasticidade cerebral, promoção da conectividade estrutural e funcional do cérebro, circuito cérebro-cerebelares e sensório-motores para melhora do controle motor e comportamentos repetitivos. Essas descobertas são a base para a Musicoterapia Neurológica de Michael Thaut, que usa o ritmo para realizar tarefas motoras complexas, ativando redes neurais compensatórias ${ }^{13}$.

A indução rítmica pode estabilizar os padrões de movimento e simplificar um plano motor, bem como estimular habilidades linguísticas, a partir da estimulação motora-oral, e melhora no potencial cognitivo, social e 
comunicativo (habilidades pessoais) consequentemente melhora na qualidade de vida ${ }^{13}$.

\section{Habilidades sociais}

Na revisão realizada por Fleury et al. ${ }^{14}$ com objetivo de associar a musicoterapia ao processo de melhora na interação e habilidades sociais de indivíduos com o Transtorno do Espectro Autismo, foi observado que a principal técnica musicoterapêutica utilizada nas intervenções com autistas é a improvisação musical, onde o paciente faz música através de instrumentos, voz ou corpo, criando ritmo, melodia, canção ou uma peça musical improvisada. Entre as abordagens destacadas, encontra-se a Musicoterapia Improvisacional, estruturada por Bruscia $(1987)^{21}$, que consiste em um conjunto de técnicas utilizadas frequentemente pelo musicoterapeuta para favorecer processos de empatia, estruturando, organizando e estimulando o processo de improvisação musical do paciente, a fim de explorar emoções e discutir questões terapêuticas.

LaGasse ${ }^{15}$ aponta a importância da música como aliada no tratamento e avaliação de pessoas com autismo e a possibilidade de ganho de diferentes habilidades. Embora a existência de diferentes abordagens de musicoterapia com enfoque nas habilidades sociais e de comunicação, encontram-se semelhanças entre elas no que diz respeito ao uso de estímulos, engajamento musical do paciente, estruturação da intervenção, interação com o paciente e o 
aprimoramento de habilidades sociais não-musicais. A música ativa redes neurais envolvidas em tarefas musicais e não-musicais semelhantes (como a fala e o canto, que ativam o giro frontal inferior esquerdo) e tem a capacidade de otimizar comportamentos alvo por meio de disparos neurais sincronizados.

Os componentes rítmicos e estruturais fornecem uma pista externa à organização, previsão, respostas apropriadas e conclusão de sequências motoras, habilidades estas que prejudicam a capacidade da pessoa envolver-se socialmente. O ritmo e a música podem fornecer uma acomodação para os déficits, pois o estímulo musical é altamente previsível e ajuda no planejamento e execução de padrões motores. Com estrutura clara baseada no tempo, a música oferece flexibilidade durante a exploração musical, as improvisações podem usar diferentes tonalidades, melodias, motivos e estruturas, facilitando a prática da interação social dentro de uma estrutura segura, meio não-verbal para praticar o envolvimento social, mudança de turno, atenção conjunta, saudação social, interação com pares e outras habilidades sociais fundamentais. A música pode fornecer percepções exclusivas dentro do processo de avaliação para indivíduos com TEA e isto deve ser considerado ${ }^{15}$.

\section{Improvisação musicoterapêutica}

Suvini ${ }^{16}$ destacou que a musicoterapia improvisada está baseada no uso de sons e músicas não estruturados, que surgem conforme as interações na própria sessão de terapia, 
tendo a sincronicidade e compartilhamento relacional como ponto de partida da interação musical, utilizando o tempo, ritmo e repetição como elementos centrais que são entendidos como reguladores tanto na música, quanto nas relações e que coloca o paciente/cliente e sua liberdade de fazer escolhas no centro de cada sessão e pode participar ativamente na construção de seu próprio caminho terapêutico.

Suvini ${ }^{16}$ correlaciona o funcionamento limitado dos neurônios-espelho a certos comportamentos autísticos e o fazer musical é uma maneira pela qual os neurôniosespelhos humanos podem ser ativados, pois é um estímulo multimodal que envolve simultaneamente aspectos visuais, auditivos e somatossensoriais, além de informações motoras. Portanto, atividades musicais baseadas em imitação e sincronização podem ser particularmente úteis no tratamento do autismo.

Ouvir música pode evocar emoções intensas e gerar ações motoras intencionais, ajudando crianças com autismo na interação, permitindo-Ihes participação e incentivo ao envolvimento social por meio da linguagem e habilidades motoras $^{16}$.

\section{Revisões sistemáticas}

Maw et al. ${ }^{17}$ pesquisaram sobre a eficácia das intervenções para crianças com autismo em idade préescolar onde a musicoterapia foi considerada a intervenção que apresentou os maiores efeitos na melhora da 
comunicação e interações sociais. Três estudos avaliaram a eficácia da intervenção musicoterapêutica usando as abordagens de desenvolvimento, comportamental e a capacidade comunicativa inerente das crianças em crianças entre três e cinco anos de idade e as principais melhorias foram nas áreas de relação pai-filho, produção de fala e linguagem, comportamentos e comunicação social não verbal. A musicoterapia se mostrou superior ao grupo controle em termos de interação social que incluiu habilidades comunicativas não verbais, habilidades de comunicação verbal e reciprocidade socioemocional. Apesar de resultados positivos sobre a intervenção musicoterapêutica, os autores destacam a falta de produção científica com amostras maiores para garantir e confirmar a eficácia da musicoterapia.

Lyra et al. ${ }^{18}$ realizaram uma revisão das revisões sistemáticas publicadas pelo Centro de Estudos Cochrane a respeito das intervenções existentes para o tratamento do autismo, onde a musicoterapia é indicada entre as intervenções não farmacológicas que parecem ser benéficas ao tratamento do autismo. Um dos estudos apresentados avaliou os efeitos da musicoterapia a curto e médio prazo por períodos de uma semana a sete meses, com resultados positivos na interação social dentro e fora do contexto terapêutico, habilidades comunicativas verbais e não verbais, qualidade na relação pai-filho e adaptação social, onde a musicoterapia se mostra melhor que a terapia "placebo". Apesar dos resultados considerados positivos, os 
autores concluem que as revisões sistemáticas sobre o tema apresentam baixa qualidade de evidência.

Na revisão de Alves et al. ${ }^{19}$ que comparou publicações nacionais e internacionais em três bases de dados a fim de identificar os tipos de pesquisas realizadas com o tema "Musicoterapia e Autismo" no período de 2016 a 2017, foram encontrados 12 artigos produzidos nos EUA, 4 na Austrália, 3 na Inglaterra, 2 no Brasil e os demais tiveram apenas um artigo em cada país. Concluiu-se que o tema ainda é pouco investigado em todo o mundo, a maioria da literatura é internacional e houve muita dificuldade no encontro de artigos, uma vez que a musicoterapia aparece de forma secundária como forma de tratamento em alguns casos. Os estudos tiveram predominância para idades de 0 a 12 anos e 13 a 18, período no qual a maioria encontra-se em atividade escolar. Os países que mais produziram são os que mais investem nas pesquisas acadêmicas e possuem fontes de incentivo para essa prática.

\section{Outras categorias}

Apesar dos biomarcadores do autismo ainda estarem em fase de mapeamento, Fiorezi et al. ${ }^{22}$ realizaram um estudo de revisão que objetivou avaliar a relação da música com os marcadores endócrinos e imunológicos associados ao autismo como a IgA (imunoglobulina, anticorpo responsável por identificar e neutralizar antígenos) e o cortisol salivar, uma vez que níveis elevados de agentes estressores afetam o desenvolvimento do sistema imunológico, fator que pode 
estar associado à diminuição de habilidades de comunicação e interação social. Fleury et al. ${ }^{14}$ apontam que o cortisol e a IgA ocorrem no organismo de forma inversamente proporcional, ou seja, quanto maior for o estresse, menor será a resposta imunológica do organismo, acarretando em danos celulares a partir do estresse oxidativo.

Os resultados indicam um aumento dos níveis de Iga e reduções significantes nos níveis de cortisol perante a estimulação musical, como foi apresentado na revisão sistemática realizada por Fancourt et al. ${ }^{23}$, acerca dos efeitos da música e aponta cinco principais modalidades de alterações nas respostas: psicológicas, fisiológicas, neurológicas, endócrinas e imunológicas, evidenciando reduções significantes nos níveis de cortisol, tanto para a escuta musical quanto para o fazer musical ativo, apontando que a música considerada relaxante foi a principal ativadora dos resultados benéficos para este biomarcador. Os autores defendem que oito dos nove estudos relatados apresentaram aumento da secreção Iga a partir da estimulação musical ${ }^{22}$.

Pensando na influência da música no cérebro e a possibilidade de visualizar as áreas e hormônios liberados diante de um estímulo sonoro-musical, Cibrian et al..$^{24}$ realizaram uma pesquisa a fim de investigar as preferências sonoras de crianças com autismo, utilizando uma faixa de Interface Cérebro-Computador (ICC) ao mesmo tempo que um grupo de 9 crianças ouviam individualmente 15 faixa sonoras, divididas entre sons naturais (vento, água e pássaros), som plano (11 repetições da mesma nota) e som 
de melodia (11 primeiras notas de uma rima infantil), tendo como resultados a indicação que as crianças com autismo ficam mais focadas ao ouvirem sons de melodia sendo tocadas por um violoncelo em tom baixo e se apresentam mais distraídas e apresentam respostas neuronais negativas perante os sons naturais apresentados. Os autores concluem que apesar das dificuldades das crianças com TEA aceitarem a faixa de ICC na cabeça, o projeto pode ser um importante instrumento para avaliação das preferências musicais entre os indivíduos com TEA.

\section{DISCUSSÃO}

Os resultados descritos anteriormente nas categorias "Intervenção", "Revisão" e "Outras Categorias" apresentaram quatro principais subcategorias da aplicação da musicoterapia no autismo: comunicação, habilidades sociais, neurofisiologia e avaliação.

\section{Comunicação}

Nos três estudos apresentados nesta seção, crianças em idade escolar entre 5 a 12 anos apresentaram melhorias na comunicação, qualidade de vida familiar, evocação de respostas musicais e instrumentais, diminuição da desatenção e de estereotipias a partir do ritmo, tendo como principal abordagem a Musicoterapia Improvisacional6-8.

O aumento da conectividade funcional entre o córtex auditivo primário bilateral e regiões subcorticais e motoras, pós-tratamento relacionou-se com a melhoria de habilidades 
de comunicação e interação. Muitas crianças com autismo que apresentam déficits na linguagem expressiva e produção da fala possuem distúrbios motores-orais, o que pode significar a necessidade de intervenções voltadas a esta demanda. O tratamento baseado no fazer musical com técnicas de imitação e sincronização, dentro das abordagens improvisacionais, facilitam o estímulo motor a partir do treinamento rítmico, que potencializa a plasticidade cerebral, comunicação e desenvolvimento de habilidades cognitivas motoras. Observando que a importância do vínculo terapêutico para o engajamento e desenvolvimento do paciente é tão importante quanto a intervenção aplicada ${ }^{6,8,13}$.

\section{Habilidades Sociais}

Dentre as diferentes abordagens e técnicas musicoterapêuticas que favorecem o desenvolvimento das habilidades sociais e de comunicação, percebe-se uma preferência para as intervenções de Musicoterapia Comportamental, Criativa (ou improvisacional) e Sensorial ${ }^{11,15}$.

As abordagens para o autismo se baseiam nos estímulos musicais que favorecem o engajamento do paciente perante o fazer musical, o que Benenzon chamaria de "Identidade Sonora" e Skinner de "Reforço", enquanto que na perspectiva neurocientífica, pode-se considerar a ativação do sistema límbico e liberação de neurotrofinas que facilitarão não somente o engajamento do paciente na sessão mas também no processo de reabilitação e aprendizagem, 
propiciando mudanças de padrões de conectividade e plasticidade cerebral ${ }^{15,25-27}$.

A música se organiza dentro do tempo de forma ordenada, criando o tão importante "ritmo". Esse elemento musical auxilia o desenvolvimento e organização neurobiológica em pessoas com autismo. Entretanto, a improvisação musical atua fornecendo a partir de estrutura segura e previsível da organização rítmica, as variações de outros elementos musicais, onde a criança pode dialogar em um contexto não-verbal os fatores que estão presentes na interação social, ativando redes neurais envolvidas em tarefas musicais e não-musicais semelhantes, facilitando a generalização ${ }^{13,15}$.

Fleury et al. ${ }^{14}$ apontam a abordagem improvisacional como principal técnica no tratamento de pessoas com autismo, indicando as técnicas de Bruscia como facilitadoras no estímulo de interação e habilidades sociais, bem como na diminuição de comportamentos estereotipados. As respostas se dão a partir da Musicoterapia Improvisacional utilizando ritmo e repetição como elementos centrais, uma vez que são reguladores nas relações, bem como a sincronicidade e compartilhamento relacional da interação musical. Este mecanismo facilita a ativação dos neurônios-espelho, promovendo a estimulação de aspectos motores, auditivos e somatossensoriais, sendo mecanismos importantes para 0 tratamento do autismo ${ }^{11,14-16 .}$

A musicoterapia, pode atuar de forma positiva quando associada a outras intervenções não-musicais, tal como a 
Terapia Lúdica, que utiliza de jogos e brinquedos como mediadores para alcançar objetivos terapêuticos, ampliando a possibilidade de generalização das brincadeiras musicais em outros contextos ${ }^{9}$.

\section{Neurofisiologia}

A intervenção musical, além de estimular o sistema de recompensa e respostas emocionais, pode ser uma importante ferramenta para a avaliação destes aspectos, bem como na promoção da conectividade cerebral, facilitando o processo de engajamento dos pacientes na terapia. A indução rítmica aumenta a conectividade cerebral e potencializa a plasticidade, ativando áreas importantes da linguagem e interação, áreas motoras, controle motor e comportamentos repetitivos e qualidade de vida ${ }^{12,13}$.

A estimulação musical diminui os níveis de cortisol salivar e aumentam a secreção do Iga, reduzindo o estresse e consequentemente aumentando a capacidade imunológica. Esse mecanismo também está relacionado às habilidades de comunicação e interação social ${ }^{22}$.

\section{Avaliação}

Levar em consideração o ISO do paciente é importante, uma vez que estimula diretamente o sistema de recompensa e facilita o engajamento do mesmo para alcançar os objetivos terapêuticos. Cibrian et al. ${ }^{24}$ apresentaram a possibilidade de investigar o gosto musical a partir da fixação de uma faixa de ICC na cabeça das crianças com TEA e 
monitorando as emoções expostas diante do estímulo musical. A grande dificuldade desta forma de avaliação se dá a aceitação da fixação da faixa de ICC pelas crianças. Isso facilitaria a investigação da Identidade Sonora no início do tratamento ${ }^{15,24}$.

\section{Revisões Sistemáticas}

A musicoterapia apresenta forte eficácia para comunicação e habilidades sociais em crianças com autismo em idade pré-escolar, sendo uma das intervenções não farmacológicas que apresentam indicações de ser benéficas, porém a produção científica sobre a musicoterapia e autismo é escassa ${ }^{17-19}$.

Todos os artigos aqui descritos apontam que existem poucos artigos e de baixa qualidade acerca da validação da musicoterapia enquanto especialidade comprovadamente eficaz no tratamento do autismo, indicando a necessidade de produção de trabalhos com amostras maiores e metodologia com maior qualidade de evidência.

\section{CONCLUSÕES}

A musicoterapia é uma forte aliada no tratamento das principais características presentes no TEA. Embora o objeto deste estudo tenha sido a musicoterapia comportamental e exista um modelo de musicoterapia com este título, as pesquisas apresentaram maior prevalência da utilização de técnicas e métodos improvisacionais para alcançar melhora nos aspectos comportamentais, experiência musical 
presente em diferentes técnicas, métodos, abordagens e modelos, ultrapassando o limiar do modelo de Musicoterapia Comportamental e evidenciando a abrangência desta especialidade para o tratamento de pessoas com autismo. Foi possível notar uma concordância acerca da influência dos elementos sonoros, especialmente o ritmo, como facilitadores para o desenvolvimento dos pacientes tanto em comunicação e habilidades sociais como na redução de estereotipias. Há evidências de que a música contribui para a regulação dos níveis de Iga e Cortisol no organismo. A musicoterapia promove a ativação dos neurônios-espelho, alterações nos padrões de conectividade cerebral e potencialização do processo de neuroplasticidade e cognição.

Há escassez de trabalhos com amostras maiores e de melhor qualidade de evidências. Também há uma falta de trabalhos que utilizem escalas validadas para avaliar a evolução musical dos pacientes, a fim de quantificar sob uma ótica musical a evolução destes indivíduos perante as intervenções musicoterapêuticas.

\section{REFERÊNCIAS}

1.Kandel ER, Schwartz JH, Jessell TM, Siegelbaum S, Hudspeth AJ. Princípios da Neurociência. 5a ed. São Paulo: AMGH, 2014.

2.Attar NMA. Effects Of Music Therapy To Enhance Verbal Skills In Children With Autism Spectrum (Dissertação). Lebanon: American University of Beirut; 2019.

https://scholarworks.aub.edu.lb/bitstream/handle/10938/21660/t-

7031.pdf?sequence $=1$

3.Junior FP. Quantos autistas há no Brasil (endereço na internet). Revista Autismo. (acessado em 2020 Jun 27). Disponível em: https://www.revistaautismo.com.br/geral/quantos-autistas-ha-no-brasil/ 4. Junior FP. Prevalência de autismo nos EUA sobe 10\%: agora é 1 para 54 (endereço na internet). Revista Autismo (acessado em 2020 Jun 27). Disponível em: 
https://www.revistaautismo.com.br/destaque/prevalencia-de-autismo-noseua-sobe-10-agora-e-1-para-

54/\# : : text=Novos\%20n\%C3\%BAmeros $\% 20$ estat $\%$ C3\%ADsticos\%20publi cados $\% 20$ hoje,cada $\% 20$ dois $\% 20$ anos $\% 20$ no $\% 20$ pa $\%$ C3\%ADs

5. Moreira S, Alcântara-Silva T, Silva D, Moreira M. Neuromusicoterapia No Brasil: Aspectos Terapêuticos Na Reabilitação Neurológica. Rev Bras Musicoterap 2012;12:18-26.

https://musicoterapia.revistademusicoterapia.mus.br/index.php/rbmt/articl e/view/265

6.Sharda M, Tuerk C, Chowdhury R, Kevin Jamey, Foster N, CustoBlanch $\mathrm{M}$, et al. Music improves social communication and auditorymotor connectivity in children with autism. TranslPsychiatry $2018 ; 8$ : 231. https://doi.org/10.1038/s41398-018-0287-3

7.Garcia MCL. La improvisación como recurso en musicoterapia: Estudio de caso único de um niño escolarizado enun aula TEA. Rev Investig Musicoterap 2018;2:35-46.

https://doi.org/10.15366/rim2018.2.005

8.Novenia MV. The Use of Music Therapy to Improve Non-Verbal Communication Skills for Children with Autism. Inter J Business Hum Soc Sci 2019;13:243-6. https://doi.org/10.5281/zenodo.2643506

9.Khanzadeh AAH, Imankhah F. The Effect of Music Therapy Along With Play Therapy on Social Behaviors and Stereotyped Behaviors of Children With Autism. J Pract Clin Psychol 2017;5:251-62.

https://doi.org/10.29252/NIRP.JPCP.5.4.251

10.Pater M, Yperen TV. The Development of Social Behavior During Music Therapy: A Child Case Report. Int J Psychiatr Res 2020;3:1-6. : https://doi.org/10.33425/2641-4317.1059

11.Bilgehan E. Profiles of the Most Preferred and the Most Effective Music Therapy Approaches being Utilized with Children with Autism Spectrum Disorders According to the Opinions of Music Therapists in the U.S. J Edu Pract 2017;8:15-128.

https://files.eric.ed.gov/fulltext/ED577837.pdf

12.Quintin EM. Music-Evoked Reward and Emotion: Relative Strengths and Response to Intervention of People With ASD. Front Neural Circ 2019;13:49. https://doi.org/10.3389/fncir.2019.00049

13. Bharathi G, Jayaramayya K, Balasubramania V, Vellingiri B. The potential role of rhythmic entrainment and music therapy intervention for individuals with autism spectrum disorders. J Exerc Rehabil 2019;15:180-6. https://doi.org/10.12965/jer.1836578.289

14.Fleury $E A B$, Santos KDS. Musicoterapia na Interação social de pessoas com TEA - Estudo de Revisão. Rev InCantare 2016;7:1-133. http://periodicos.unespar.edu.br/index.php/incantare/article/view/1754

15. LaGasse $A B$. Social outcomes in children with autism spectrum disorder: a review of music therapy outcomes. Patient Relat Outcome Meas 2017;8:23-32. https://doi.org/10.2147/PROM.S106267

16.Suvini FM. The application of improvisational music therapy in autism. Life Res 2019;2:52-8. https://doi.org/10.12032/life2019-0425$\underline{001}$

17.Maw SS, Haga C. Effectiveness of cognitive, developmental, and behavioural interventions for Autism Spectrum Disorder in preschool- 
aged children: A systematic review and meta-analysis. Heliyon 2018;4:1-20. https://doi.org/10.1016/j.heliyon.2018.e00763

18.Lyra L, Rizzo LE, Sunahara CS, Pachito DV, Latorraca COC, Martimbianco ALC, et al. What do Cochrane systematic reviews say about interventions for autism spectrum disorders? Sao Paulo Med J 2017;135:192-201.

https://doi.org/10.1590/1516-

\subsubsection{7}

19. Alves TAT, Ribeiro T, Theodório DP. Musicoterapia: Intervenção Em Individuos Com Transtorno Do Espectro Autista (TEA) - Revisão Sistemática. Rev Cient UMC 2018;3:1-4.

http://seer.umc.br/index.php/revistaumc/article/view/473/365

20.Trevarthen C, Daniel S. Disorganized rhythm and synchrony: early signs of autism and Rett syndrome. Brain Dev 2005;27(Suppl 1):S2534. https://doi.org/10.1016/j.braindev.2005.03.016

21. Bruscia KE. Improvisational Models of Music Therapy. Illinois: Charles C Thomas Pub Ltd; 1987.

22.Fiorezi JN, Franke SIR, Prá D, Garcia EL, Renner JDP. Os efeitos da música em biomarcadores de estresse, imunológicos e comportamentais em portadores do espectro autista. UNISC 2017;18:1-8. https://doi.org/10.17058/cinergis.v18i0.11174

23. Fancourt D, Ockelford A, Belai A. The psychoneuroimmunological effects of music: a systematic review and a new model. Brain Behav Immun 2014;36:15-26. https://doi.org/10.1016/j.bbi.2013.10.014

24. Cibrian FL, Mercado J, Escobedo L, Tentori M. A Step towards Identifying the Sound Preferences of Children with Autism. In: Proceedings of the 12th EAI International Conference on Pervasive Computing Technologies for Healthcare. Pervasive Health 2018:15867. https://doi.org/10.1145/3240925.3240958

25.Godoy DA. Musicoterapia, profissão e reconhecimento: uma questão de identidade, no contexto social brasileiro. Rev Bras Musicoterap 2014;16:6-25.

http://www. revistademusicoterapia.mus.br/wp-content/uploads/2016/10/1MUSICOTERAPIA-PROFISS\%C3\%830-E-RECONHECIMENTO-UMA-

QUEST\%C3\%830-DE-IDENTIDADE-NO-CONTEXTO-SOCIAL-

BRASILEIRO.pdf

26.Rezende E. O que é reforço positivo e negativo (endereço na Internet). 2017 (acessado em 2020 Nov 18). Disponível em: https://www.psicoedu.com.br/2017/03/reforco-positivo-negativoexemplo.html

27.Silva L. Neuroplasticidade e música: um estudo sobre as neurociências e a educação musical. In: Anais do II Congresso Interdisciplinar de Pesquisa, Iniciação Científica e Extensão do Centro Universitário Metodista Izabela Hendriz, 2017, p.943-54. 